\title{
miR-186, a serum microRNA, induces endothelial cell apoptosis by targeting SMAD6 in Kawasaki disease
}

\author{
RONGZHOU WU ${ }^{1 *}$, DANPING SHEN ${ }^{1 *}$, HARESHWAREE SOHUN $^{1}$, DONGHUI GE $^{1}$, XIANDA CHEN $^{1}$, \\ XULIANG WANG ${ }^{1}$, RUIYAO CHEN ${ }^{1}$, YUQING WU ${ }^{2}$, JINGJING ZENG ${ }^{1}$, \\ XING RONG ${ }^{1}$, XIAOPING SU ${ }^{1}$ and MAOPING CHU ${ }^{1}$ \\ ${ }^{1}$ Children's Heart Center, Institute of Cardiovascular Development and Translational Medicine, \\ The Second Affiliated Hospital \& Yuying Children's Hospital of Wenzhou Medical University, Wenzhou, Zhejiang 325027; \\ ${ }^{2}$ Wenzhou Medical University, Wenzhou, Zhejiang 325035, P.R. China
}

Received October 17, 2017; Accepted January 16, 2018

DOI: $10.3892 / \mathrm{ijmm} .2018 .3397$

\begin{abstract}
Kawasaki disease (KD) is an acute, self-limited vasculitis that predominantly affects medium-sized arteries, particularly the coronary arteries. Recent studies have indicated that microRNAs are involved in many diseases, including KD. However, the detailed mechanism remains unclear. The aim of the present study was to explore the role of miR-186 in $\mathrm{KD}$ and potentially discover a new target for KD treatment. The results demonstrated that miR-186 was upregulated in serum from patients with KD and KD serum could increase miR-186 transcript levels in endothelial cells (HUVECs). Overexpression of miR-186 mimic induced HUVEC apoptosis through mitogen-activated protein kinase (MAPK) activation by targeting and inhibiting SMAD family member 6 (SMAD6). Furthermore, KD serum induced HUVEC apoptosis through miR-186. In conclusion, the present results suggested that KD serum-associated miR-186 has an essential role in endothelial cell apoptosis by activating the MAPK pathway through targeting the SMAD6 gene.
\end{abstract}

\section{Introduction}

Kawasaki disease (KD) is an acute, systemic vasculitis that is the leading cause of acquired heart disease in children in the

Correspondence to: Professor Maoping Chu or Professor Xiaoping Su, Children's Heart Center, The Second Affiliated Hospital and Yuying Children's Hospital, Institute of Cardiovascular Development and Translational Medicine, Wenzhou Medical University, 105 Xueyuan Road, Wenzhou, Zhejiang 325027, P.R. China

E-mail: chmping@hotmail.com

E-mail: bloooge@163.com

*Contributed equally

Key words: Kawasaki disease, microRNA, miR-186, endothelial cell, mitogen-activated protein kinase pathway, SMAD family member 6 developed world (1). Vascular inflammation ultimately leads to coronary artery abnormalities (CAA), including aneurysm, myocardial fibrosis, and aortic root dilatation $(2,3)$. Within the first 10 days after onset of fever, CAA develops in $25 \%$ of untreated patients and in 3-5\% of patients treated with intravenous immunoglobulin (IVIG) (4). Histological examination of tissues from KD autopsies revealed significant vascular lesions. Regressive changes, such as degeneration, desquamation or necrosis, were observed in the endothelial cells of the damaged artery (5). Endothelial cell injuries appear to cause edematous and inflammatory cell infiltration in the subendothelial space, which eventually leads to coronary artery anomalies $(6,7)$. A follow-up study of KD demonstrated that a subset of patients had persistent endothelial injury many years following KD, both with and without CAA (8). Much effort has been spent on the study of the biological mechanism of KD, and the clinical events of arteritis are well-characterized (9). However, the molecular mechanisms underlying the development of KD remain poorly understood.

MicroRNAs (miRNAs) are a class of endogenous, small, noncoding RNAs that control gene expression eitherby inducing transcript degradation or by blocking translation (10). Recently, miRNAs have been reported to be present in human plasma in a remarkably stable form, and to be involved in the regulation of diverse developmental and pathological processes $(11,12)$. Previous studies have demonstrated that miRNAs act as new promising diagnostic and prognostic biomarkers for various diseases, including KD. Yun et al (13) and Shimizu et al (14) demonstrated that miRNAs were significantly upregulated in the sera or peripheral blood of patients with KD compared with healthy controls. They concluded that the possible pathways of these miRNAs predominantly involved inflammatory responses and the transforming growth factor (TGF)- $\beta$ pathway. Rowley et al (15) used a custom quantitative nuclease protection assay to reveal a set of miRNAs that appear to be dysregulated in KD coronary arteries. Rong et al (16) reported that miR-92a had a relatively high degree of sensitivity and specificity in predicting the incidence of coronary artery disease. It is worth mentioning that He et al (17) demonstrated that the Kruppel-like factor 4/miR-483/connective tissue growth factor pathway may be useful in the evaluation of the 
Table I. Characteristics of the clinical index independent validation cohort in different groups.

\begin{tabular}{lcccc}
\hline Groups & Healthy control & Febrile children & Convalescent KD & Acute KD \\
\hline Number & 25 & 17 & 11 & 21 \\
Male & $17(68 \%)$ & $10(59 \%)$ & $6(55 \%)$ & $16(76 \%)$ \\
Female & $8(32 \%)$ & $7(41 \%)$ & $5(45 \%)$ & $5(24 \%)$ \\
Age (months) & $50.4 \pm 28.35$ & $49.27 \pm 32.78$ & $30.68 \pm 27.18$ & $32.95 \pm 16.18$ \\
WBC $\left(\right.$ x $\left.10^{9} / \mathrm{l}\right)$ & $7.98 \pm 2.47$ & $10.86 \pm 6.04$ & $8.78 \pm 3.18$ & $16.16 \pm 6.33$ \\
PLT $\left(\mathrm{x} 10^{9}\right)$ & $331.24 \pm 76.75$ & $262.4 \pm 68.1$ & $529.18 \pm 212.96$ & $378.47 \pm 102.88$ \\
CRP $(\mathrm{mg} / \mathrm{l})$ & $\mathrm{md}$ & $20.85 \pm 18.04$ & $3.95 \pm 2.94$ & $78.61 \pm 57.41$ \\
ALT $(\mathrm{U} / \mathrm{l})$ & $16.11 \pm 9.62$ & $15.47 \pm 7.95$ & $100.1 \pm 131.6$ & $87.13 \pm 116.15$ \\
AST $(\mathrm{U} / \mathrm{l})$ & $33.33 \pm 9.8$ & $33.4 \pm 10.74$ & $47.9 \pm 21.83$ & $37.5 \pm 43.4$ \\
NT-proBNP $(\mathrm{pg} / \mathrm{ml})$ & $\mathrm{md}$ & $\mathrm{md}$ & $179.73 \pm 133.4$ & $1,657.2 \pm 3,268$ \\
D-dimmer $(\mu \mathrm{g} / \mathrm{ml})$ & md & md & $0.9 \pm 0.46$ & $1.41 \pm 0.86$
\end{tabular}

KD, Kawasaki disease; WBC, white blood cell; PLT, platelet; ALT, glutamic pyruvic transaminase; AST, glutamic oxaloacetic transaminase; NT-proBNP, N-terminal pro natriuretic peptide type B; md, missing data.

pro-inflammatory and pro-endothelial-to-mesenchymal transition status of KD patients. Recently, Chu et al (18) determined the role of blood miR-223 in KD and KD-induced injuries in vascular endothelial cells, as well as the mechanisms involved.

In our previous study, differentially expressed miRNAs were profiled in KD serum (18). The results of the previous study demonstrated that miR-186 is among the highly expressed miRNAs in the serum of a patient with KD. Recent studies on miR-186 have focused on its role in carcinogenesis, by regulating cell proliferation, apoptosis and metastasis $(19,20)$. However, very little is known about the role and underlying mechanism of miR-186 in KD. In the present study, miR-186 was demonstrated to be enriched in acute KD serum compared with healthy controls and febrile controls, and its expression was decreased to normal levels in serum from convalescent KD (3 weeks following fever onset). Overexpression of miR-186 mimic promoted apoptosis in human umbilical vein endothelial cells (HUVECs). Furthermore, miR-186 overexpression promoted mitogen-activated protein kinase (MAPK) activation in HUVECs by targeting and inhibiting SMAD family member 6 (SMAD6). By contrast, miR-186 inhibitor limited the effect of KD serum on HUVEC apoptosis. The present results suggested that miR-186 present in KD serum may act as a treatment target for the disease.

\section{Materials and methods}

Subjects. The demographic and clinical characteristics of the subjects involved in the present study are presented in Table I. All patients diagnosed with KD had fever for at least three days and met at least four of the five clinical criteria for KD (rash, conjunctival injection, cervical lymphadenopathy, oral mucosal changes, and changes in the extremities), or three of the five criteria along with coronary artery abnormalities documented by echocardiogram (21). Patients diagnosed with acute upper respiratory infection and herpangina, which mimic many of the clinical and laboratory findings in acute $\mathrm{KD}$, were prospectively enrolled as febrile control subjects. In the present study, the sera of 21 acute KD children, 25 healthy controls, 17 febrile children and 11 convalescent KD children were collected from the second affiliated Hospital of WenZhou Medical University (WenZhou, China) between May 2014 and May 2016. All serum samples were stored at $-80^{\circ} \mathrm{C}$ until usage. The study protocol was approved by The Second Affiliated Hospital of WenZhou Medical University Ethical Committee.

Cell culture and treatment. Human umbilical vein endothelial cells (HUVECs) were purchased from American Type Culture Collection (Manassas, VA, USA) and cultured in Roswell Park Memorial Institute 1640 medium (Gibco; Thermo Fisher Scientific, Inc., Waltham, MA, USA) supplemented with $10 \%$ (vol/vol) fetal bovine serum (FBS; Invitrogen; Thermo Fisher Scientific, Inc., Waltham, MA, USA). Cells were maintained at $37^{\circ} \mathrm{C}$ in a humidified atmosphere with $5 \% \mathrm{CO}_{2}$. HUVECs were transiently transfected with miR-186 mimics, miR-186 inhibitors or negative control oligonucleotides using Lipofectamine 2000 reagent (Invitrogen; Thermo Fisher Scientific, Inc.), according to the manufacturer's instructions. RNA oligos were chemically synthesized and purified by Ribo Bio Co., Ltd. (Guangzhou, China). The sequence of human miR-186 mimic was 5'-CAA AGAAUUCUCCUUUUGGGCU-3'. The sequence of the human miR-186 inhibitor was 5'-GUUUCUUAAGAGGAA AACCCGA-3'. The negative control oligonucleotide for the miRNA mimic was 5'-UUUGUACUACACAAAAGUACU G-3'. Cells were treated for the indicated time with TGF- $\beta 1$ $(5 \mathrm{ng} / \mathrm{ml})$ after $24 \mathrm{~h}$ of transfection with miR-186 mimic $(10 \mathrm{ng} / \mathrm{ml})$. In other experiments, cells were treated for $24 \mathrm{~h}$ with healthy control serum, KD serum alone, or KD serum with miR-186 inhibitor (30 ng/ml).

RNA isolation and reverse transcription-quantitative polymerase chain reaction $(R T-q P C R)$. Total RNA was isolated from $200 \mu 1$ serum specimen or cells using TRIzol reagent (Thermo Fisher Scientific, Inc.), according to the manufacturer's protocol. The extracted RNA was reverse transcribed 
Table II. Primers used in the present study.

\begin{tabular}{|c|c|}
\hline Name & Primer sequence $\left(5^{\prime}-3^{\prime}\right)$ \\
\hline U6 RT & AACGCTTCACGAATTTGCGT \\
\hline U6 F & CTCGCTTCGGCAGCACA \\
\hline U6 R & GTGCAGGGTCCGAGGT \\
\hline GAPDH F & AACTCTGGTAAAGTGGATATTG \\
\hline GAPDH R & GGTGGAATCATATTGGAACA \\
\hline Cel-miR-39 RT & $\begin{array}{l}\text { GTCGTATCCAGTG CAGGGTCC } \\
\text { GAGGTATTCGCACTGGATACGA } \\
\text { CCAAGCT }\end{array}$ \\
\hline Cel-miR-39 F & GCCGCTCACCGGGTGTAAATC \\
\hline Cel-miR-39 R & GTGCAGGGTCCGAGGT \\
\hline SMAD6 F & CTGGAGTTGTTGAGCAGCC \\
\hline SMAD6 R & GTGCGTCTTTCTTGTTTTGTCC \\
\hline SMAD6(Luci)WT F & $\begin{array}{l}\text { AAACTAGTGCACTTTGGCTTATA } \\
\text { ATTCTTTCAATACAG }\end{array}$ \\
\hline SMAD6(Luci)WT R & $\begin{array}{l}\text { GGAAGCTTCAAACCCAGGCTTT } \\
\text { TCCACC }\end{array}$ \\
\hline SMAD6(Luci)Mut F & $\begin{array}{l}\text { AAACTAGTGCACTTTGGCTTA } \\
\text { TATAAGAATCAATACAG }\end{array}$ \\
\hline SMAD6(Luci)Mut R & $\begin{array}{l}\text { GGAAGCTTCAAACCCAGGCTTT } \\
\text { TCCACC }\end{array}$ \\
\hline miR-186 RT & $\begin{array}{l}\text { GTCGTATCCAGTGCAGGGTCCG } \\
\text { AGGTATTCGCACTGGATACGAC } \\
\text { AGCCCA }\end{array}$ \\
\hline miR-186 F & GCCGCCAAAGAATTCTCCTTT \\
\hline miR-186 R & GTGCAGGGTCCGAGGT \\
\hline
\end{tabular}

RT, reverse transcription; F, forward primer; R, reverse primer; SMAD6, SMAD family member 6; Luci, luciferase reporter; WT, wild-type; Mut, mutation; cel-miR-39, Caenorhabditis elegans miR-39.

into cDNA using ReverTra Ace qPCR RT kit (Toyobo Co., Ltd., Osaka, Japan). qPCR was performed using Power SYBR-Green PCR Master Mix (Thermo Fisher Scientific, Inc.) on an Bio-Rad CFX 96 Real-Time PCR System (Bio-Rad Laboratories, Inc., Hercules, CA, USA). Briefly, samples were incubated at $95^{\circ} \mathrm{C}$ for $10 \mathrm{~min}$ for an initial denaturation, followed by $40 \mathrm{PCR}$ cycles of at $95^{\circ} \mathrm{C}$ for $15 \mathrm{sec}$ and then $60^{\circ} \mathrm{C}$ for $1 \mathrm{~min}$. The specificity of SYBR Green based PCR reaction was validated by post-amplification melting curve analysis. For cell samples, U6 was used as an internal control for miRNA template normalization and GADPH was used for other template normalizations. For serum samples, the internal control was a spiked-in control (cel-miR-39). Fluorescent signals were normalized to the internal reference, and the threshold cycle was set within the exponential phase of the PCR. miRNA and mRNA expression levels were determined using the $2^{-\triangle \Delta C T}$ method (22). The RT primer and forward and reverse primer pairs for miR-186 were designed by Generay Biotech Co., Ltd. (Shanghai, China). Primer sequences are listed in Table II.
Western blotting. The cells were lysed with RIPA buffer containing protease and phosphatase inhibitors (complete ULTRA tablets; Roche Molecular Diagnostics, Branchburg, NJ, USA). Following centrifugation (12,000 x g, $\left.4^{\circ} \mathrm{C}, 30 \mathrm{~min}\right)$, the supernatant was collected and quantified. The proteins were quantified using a BCA Kit (Beyotime Institute of Biotechnology, Jiangsu, China). A total of $30 \mu \mathrm{g}$ of protein was loaded per lane in the $10 \%$ gel and separated by SDS-PAGE, then transferred to nitrocellulose membranes. After blocking with $5 \%$ non-fat milk at room temperature for $2 \mathrm{~h}$, the membranes were probed with antibodies against SMAD6 (cat. no. sc-7004; Santa Cruz Biotechnology, Dallas, TX, USA), TNF receptor-associated factor 6 (TRAF6; cat. no. ab40675; Abcam, Cambridge, MA, USA), phosphorylated (p-) TGF- $\beta$-activated kinase 1 (TAK1)/total TAK1 (cat. no. 5206, cat. no. 9339; Cell Signaling Technology, Danvers, MA, USA), p-P38/total P38 MAPK (cat. no. 4511, cat. no. 8690; Cell Signaling Technology), p-c-Jun N-terminal kinase (JNK)/total JNK (cat. no. 4668, cat. no. 9252; Cell Signaling Technology), BCL2 associated X (cat. no. 2772, Bax; Cell Signaling Technology), BCL2 apoptosis regulator (cat. no. 3498, Bcl-2; Cell Signaling Technology), or cleaved Caspase-3 (cat. no. 9664, Cell Signaling Technology) overnight at $4^{\circ} \mathrm{C}$. Membranes were then incubated with a fluorescence-labeled secondary antibody at room temperature for $2 \mathrm{~h}$. GAPDH antibody (cat. no. 5174, Cell Signaling Technology) was used as a loading control. Secondary antibodies included HRP-conjugated against rabbit (cat. no. 7074, Cell Signaling Technology, Inc.) and Rabbit anti-Goat peroxidase conjugated $\mathrm{H}+\mathrm{L}$ (cat. no. BL004A, Zhihong Biotechnology Co., Ltd., Hefei, China). Immunoblots were visualized using Biorad ChemiDoc XRS+ with Image Lab Software (version 5.2.1, Hangzhou Baocheng Biotechnology Co., Ltd., Hangzhou, China). Densitometry of Western blots was quantified with Image Lab software (version 5.2.1, Hangzhou Baocheng Biotechnology Co., Ltd.).

Luciferase reporter assay. A luciferase reporter construct was generated by cloning the human SMAD6 mRNA sequence into the pMIR-Report vector (Ambion; Thermo Fisher Scientific, Inc.). Wild-type or mutant SMAD6 mRNA fragments were amplified and cloned into the luciferase reporter via SpeI and HindIII sites. All the primers are listed in Table II. HUVECs plated in a 48 -well plate were co-transfected with $10 \mathrm{nM}$ miRNA mimics or negative control oligonucleotides, $50 \mathrm{ng}$ of firefly luciferase reporter and $10 \mathrm{ng}$ of pRL-TK (Promega Corporation, Madison, WI, USA) using the JetPRIME reagent (Polyplus-transfection). Cells were collected $36 \mathrm{~h}$ after last transfection and analyzed using Dual-Luciferase Reporter Assay System (Promega Corporation).

Terminal deoxynucleotidyl transferase dUTP nick end labeling (TUNEL) assay. TUNEL staining was used to detect DNA fragmentation of individual cells using a TUNEL fluorescence fluorescein isothiocyanate (FITC) kit (Roche Diagnostics, Indianapolis, IN, USA). HUVECs grown on coverslips were fixed with $4 \%$ paraformaldehyde at $37^{\circ} \mathrm{C}$ for $1 \mathrm{~h}$ followed by permeabilization with $0.1 \%$ Triton $\mathrm{X}-100$. Then, cells were incubated with TUNEL reaction mixture at $37^{\circ} \mathrm{C}$ for $1 \mathrm{~h}$. The stained cells were examined under a fluorescence microscope. 


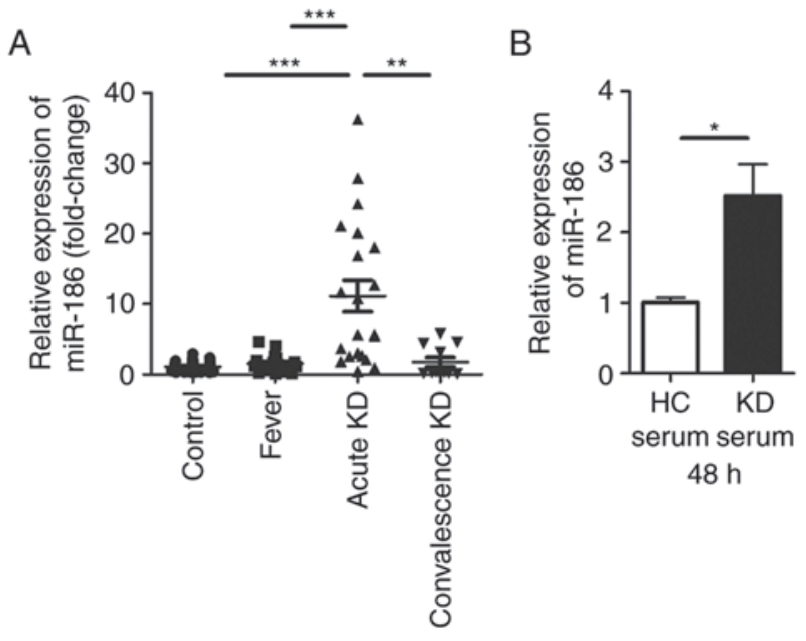

Figure 1. Serum miR-186 levels are increased in patients with KD. (A) miR-186 levels were determined in serum samples from 21 acute KD patients, 25 healthy control participants, 17 febrile control participants and 11 convalescence KD patients. (B) Expression of miR-186 was measured in HUVECs by reverse transcription-quantitative polymerase chain reaction at $48 \mathrm{~h}$ following culture with $20 \%$ serum from KD patients or healthy controls $(\mathrm{n}=3)$. Data are presented as the mean \pm standard error of the mean. ${ }^{*} \mathrm{P}<0.05$, ${ }^{* *} \mathrm{P}<0.01$, and ${ }^{* * * *} \mathrm{P}<0.001$, with comparisons indicated by lines. KD, Kawasaki disease; HUVECs, human umbilical vein endothelial cells; HC, healthy control.

Statistical analysis. Results are presented as the mean \pm standard error of the mean. Differences among groups were analyzed using one-away analysis of variance accompanied with Turkey multiple-comparisons test. Two-tailed Student's t-test was used for comparison between two groups. Statistical analyses were performed with GraphPad Prism version 5.0 (GraphPad Software, Inc., La Jolla, CA, USA). $\mathrm{P}<0.05$ was considered to indicate a statistically significant difference.

\section{Results}

miR-186 is upregulated in KD serum and in HUVECs stimulated with KD serum. Although our previous study revealed that miR-186 was differentially expressed in KD serum compared with healthy children (18), the function of miR-186 in KD remained unclear. In the present study, RT-qPCR was used to confirm that miR-186 was significantly upregulated in KD serum compared with serum from healthy children as well as serum from febrile children (Fig. 1A). The results demonstrated that the expression of miR-186 in the KD serum was $\sim 10$-fold higher compared with healthy controls and febrile children. In serum sampled from children with convalescence KD, miR-186 exhibited a tendency of decreasing back to normal levels (Fig. 1A).

$\mathrm{KD}$ is an acute rash autoimmune disease, primarily affecting blood vessels and inducing systemic vasculitis (1). As previously reported, endothelial damage is the first step in the development of coronary artery aneurysm during the course of KD $(6,21)$. Since previous studies of KD have reported that HUVECs can be used to study the vascular endothelium $(23,24)$, these cells were used as the in vitro model in the present study. In order to examine whether KD serum could

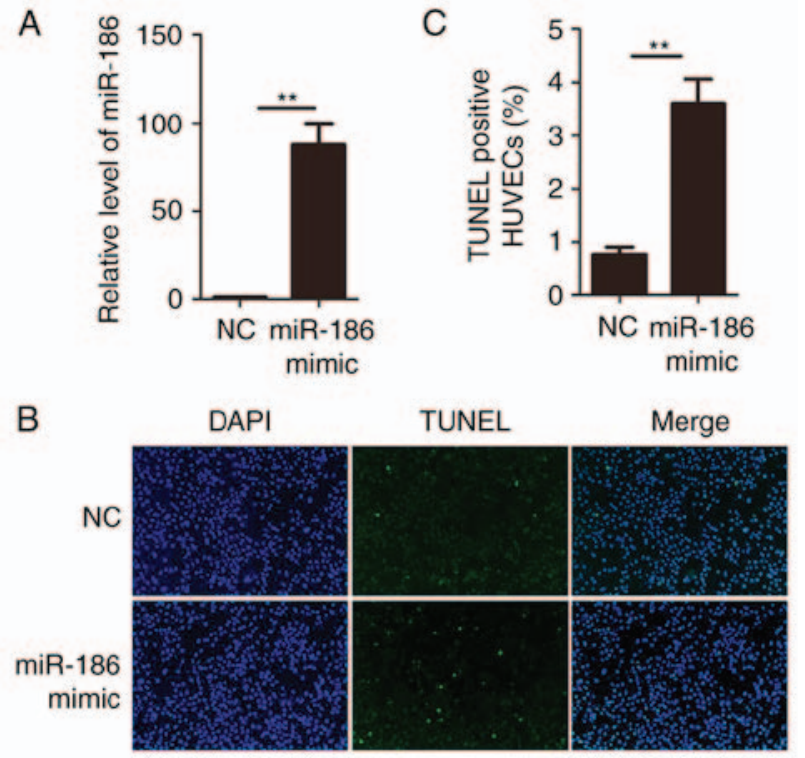

Figure 2. miR-186 overexpression induces HUVEC apoptosis. (A) HUVECs were transfected with miR-186 mimics or negative control oligonucleotides. miR-186 expression was quantified by reverse transcription-quantitative polymerase chain reaction $(\mathrm{n}=3)$. (B) The effect of miR-186 overexpression on HUVEC apoptosis induced by $\mathrm{H}_{2} \mathrm{O}_{2}(100 \mu \mathrm{mol} / 1)$ for $3 \mathrm{~h}$ was determined by TUNEL assay. Representative micrographs exhibiting cell images are shown at x100 magnification. (C) The \% of TUNEL + cells was calculated in 10 random fields per slide $(n=3)$. Data are presented as the mean \pm standard error of the mean. ${ }^{* *} \mathrm{P}<0.01$. HUVECs, human umbilical vein endothelial cells; NC, negative control; TUNEL, terminal deoxynucleotidyl transferase dUTP nick end labeling.

increase miR-186 in endothelial cells, HUVECs were cultured with $20 \%$ KD serum or $20 \%$ healthy control (HC) serum. To prevent the immune response of these sera, they were inactivated in a $56^{\circ} \mathrm{C}$ water bath for $30 \mathrm{~min}$ prior to experiments. miR-186 levels were then determined in HUVECs $48 \mathrm{~h}$ following $\mathrm{HC}$ or KD serum incubation. A significant increase of miR-186 levels was identified in cells cultured with KD serum, but not in cells cultured with HC serum (Fig. 1B). As a result, it was speculated that miR-186 could affect endothelial cells in the pathological process of KD.

Overexpression of miR-186 induces HUVEC apoptosis. Since miR-186 was upregulated in KD serum and KD serum could upregulate miR-186 in HUVECs, gain-of-function studies were performed in HUVECs. miR-186 mimics or negative control oligonucleotides were transiently transfected into HUVECs. Expression of miR-186 was determined by RT-qPCR. The results confirmed that transfection of miR-186 mimic significantly increased its expression in HUVECs (Fig. 2A). The functional role of miR-186 was then examined on HUVECs by performing TUNEL assays. Overexpression of miR-186 in HUVECs resulted in a significant increase of cell apoptosis compared with the negative control oligonucleotide transfection (Fig. 2B and C). These data suggested that miR-186 modulated HUVEC viability and that overexpression of miR-186 promoted HUVEC apoptosis.

miR-186 directly targets SMAD6. To understand the mechanisms by which miR-186 induces endothelial cell apoptosis, several miRNA target prediction 
A

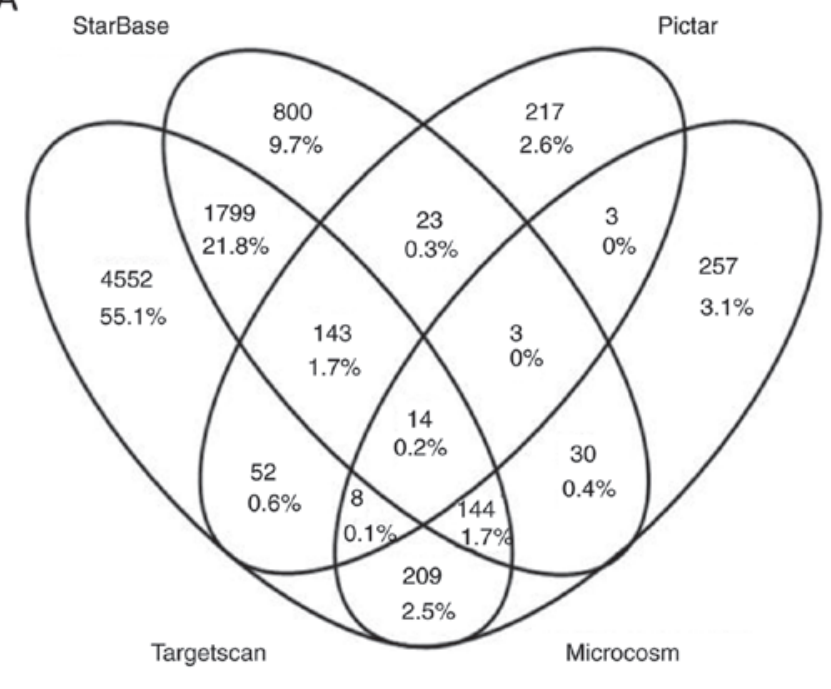

C

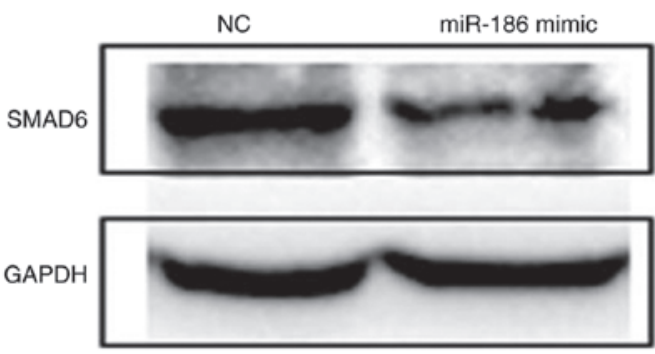

B
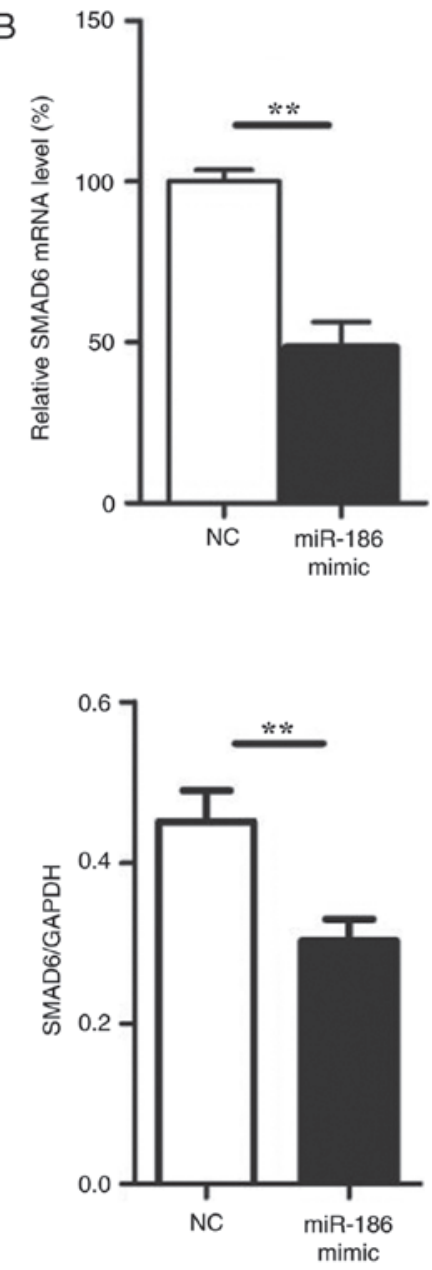

D 2769-2792 SMAD6 mRNA(WT): 5'-ACGCACUUUGGCUUAUAAUUCUUU-3' miR-186: 3'-UCGGGUUUUCCUCUUAAGAAAC-5 2769-2792 SMAD6 mRNA(MUT): 5'-ACGCACUUUGGCUUAUÁAAAGA'́u-3' $\mathrm{E}$

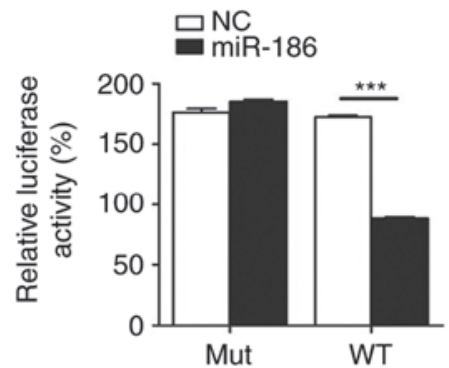

Figure 3. miR-186 directly targets SMAD6. (A) Venn diagram of the results from the gene target prediction algorithms. (B) Effect of miR-186 mimic overexpression on the endogenous SMAD6 mRNA levels $(n=3)$. (C) Effect of miR-186 mimic overexpression on the endogenous SMAD6 protein levels $(\mathrm{n}=3$ ). (D) Schematic of the predicted miR-186 binding sequence on the SMAD6 mRNA 3'-UTR. Luciferase reporter construct was made by cloning the human SMAD6 mRNA sequence into pMIR-Report construct. Wild-type or mutant SMAD6 mRNA fragments (from 2,769 to 2,792) were amplified and cloned into the luciferase reporter via SpeI and HindIII sites. (E) HUVECs were co-transfected with the reporter constructs bearing the wild-type and mutant SMAD6 sequences as indicated in Fig. 3D, and with miR-186 mimics or negative control oligonucleotides. After $36 \mathrm{~h}$, firefly luciferase activity was measured and normalized to Renilla luciferase activity. Data are presented as the mean \pm standard error of the mean. ${ }^{* *} \mathrm{P}<0.01$, and ${ }^{* * *} \mathrm{P}<0.001$, with comparisons indicated by lines. SMAD6, SMAD family member 6; UTR, untranslated region; HUVECs, human umbilical vein endothelial cells; NC, negative control; WT, wild-type; MUT, mutant.

algorithms, including Microcosm (http://www.ebi.ac. uk/enright-srv/microcosm/htdocs/targets/v5/) (25), starBase (http://starbase.sysu.edu.cn/) (26), Pictar (http://pictar.mdcberlin.de/) (27) and TargetScan (http://www.targetscan.org/) (12) were used to identify the potential target genes of miR-186. A total of 14 target genes were predicted by all four prediction algorithms used in the present study (Fig. 3A; Table III). Among these genes, SMAD6 was also demonstrated to be targeted by miR-186 from experimental data in the DIANATarBase v7.0 database (http://diana.imis.athenainnovation.gr/DianaTools/index.php?r=tarbase/) (28), and therefore we focused on SMAD6. To determine whether 
A

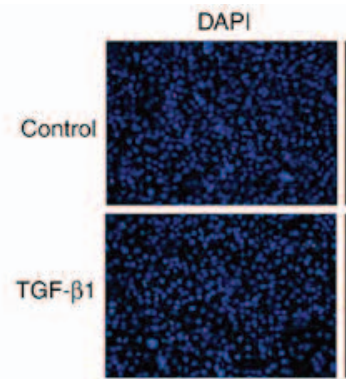

TUNEL
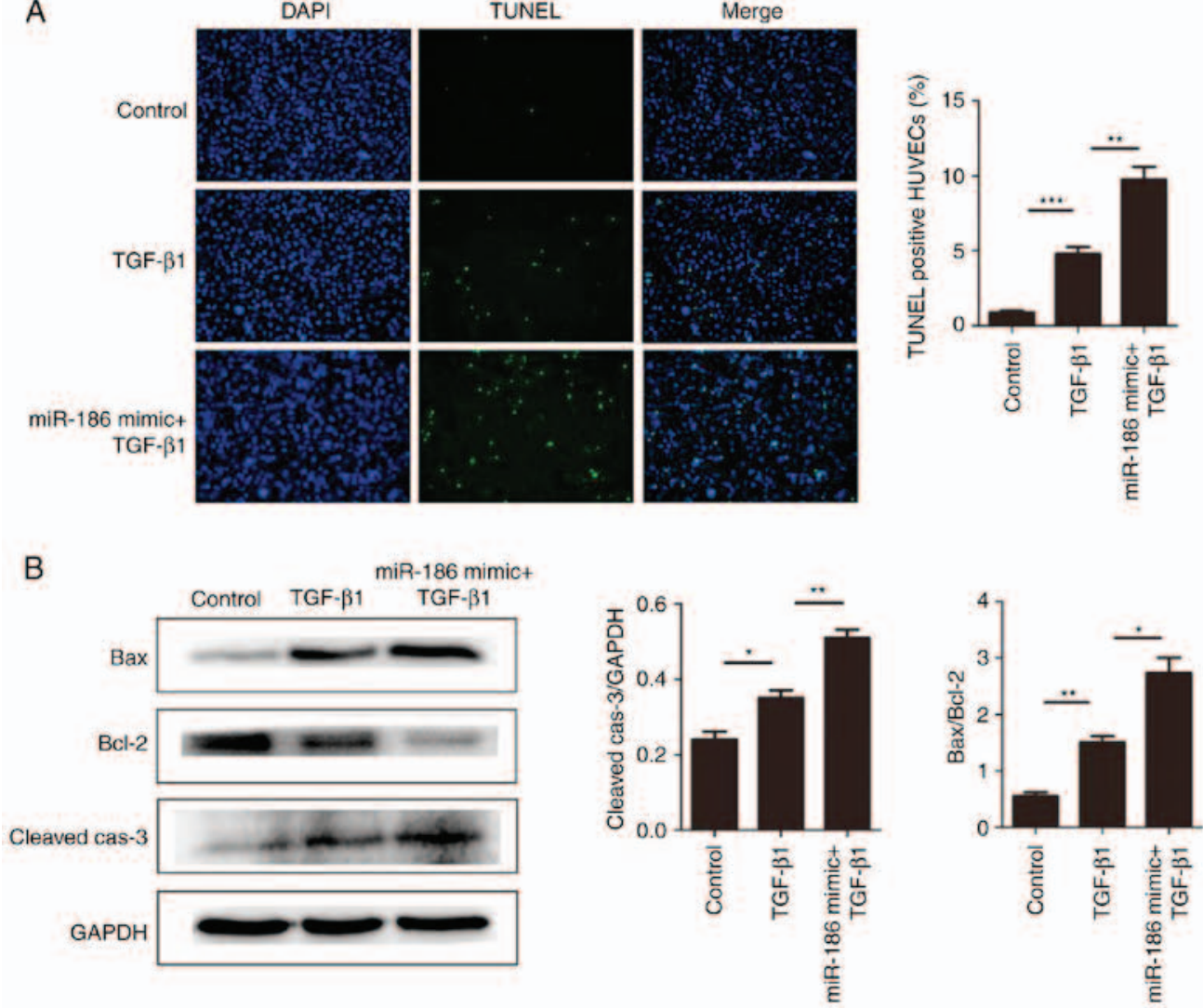

C
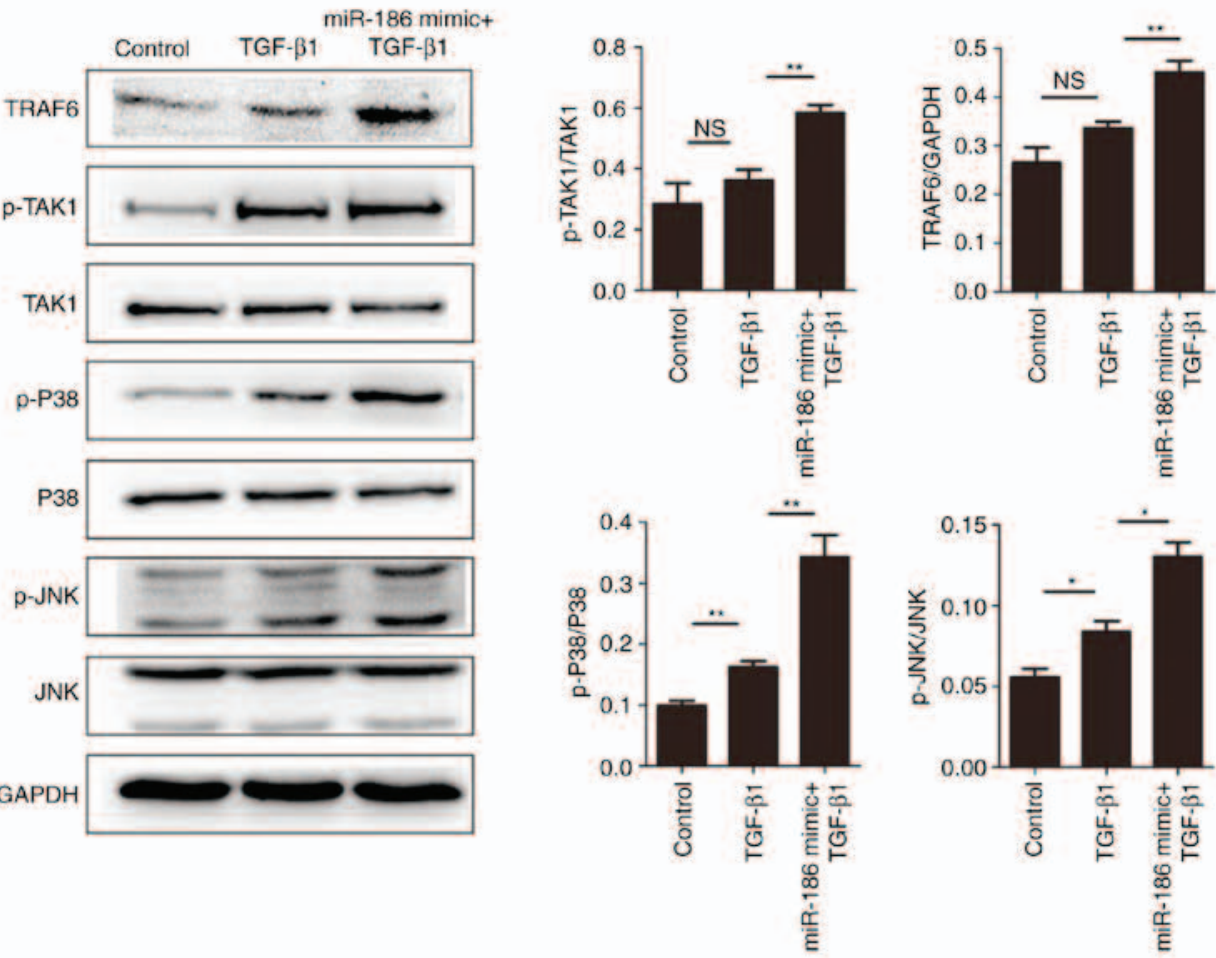

Figure 4. miR-186 overexpression enhances TGF- $\beta 1$-mediated apoptosis in HUVECs. (A) The effect of miR-186 overexpression on HUVEC apoptosis induced by TGF- $\beta 1(5 \mathrm{ng} / \mathrm{ml})$ was measured by TUNEL assay. Representative images are shown at x100 magnification and quantification of $\%$ of TUNEL + cells were calculated in 10 random fields per slide $(n=4)$. (B) miR-186 overexpression increased the TGF- $\beta 1$-mediated expression of apoptosis-related proteins Representative blots and quantification is shown. The expression levels of Bax/Bcl-2 in the TGF- $\beta 1$ group were higher compared with the control group $(\mathrm{P}=0.02)$, but in the TGF- $\beta 1+$ miR-186 mimic transfection group were higher compared with the TGF- $\beta 1$ alone group $(\mathrm{P}=0.006)$. The expression levels of cleaved-caspase-3 (normalized to GAPDH) in the TGF- $\beta 1$ group were higher compared with the control group $(\mathrm{P}=0.023)$, but in the TGF- $\beta 1+$ miR-186 mimic transfection group were higher compared with the TGF- $\beta 1$ alone group $(\mathrm{P}=0.004)$. (C) Expression levels of MAPK pathway-related proteins were detected by western blotting in HUVECs induced by TGF- $\beta 1$ with or without miR-186 mimic transfection. Compared with the TGF- $\beta 1$ alone group, TGF- $\beta 1+$ miR-186 mimic transfection significantly activated the MAPK pathway $(\mathrm{P}<0.05)$. Data are presented as the mean \pm standard error of the mean. ${ }^{*} \mathrm{P}<0.05,{ }^{* *} \mathrm{P}<0.01$, and ${ }_{* * * *} \mathrm{P}<0.001$, with comparisons indicated by lines. TGF, transforming growth factor; HUVECs, human umbilical vein endothelial cells; TUNEL, terminal deoxynucleotidyl transferase dUTP nick end labeling; Bcl-2, BCL2 apoptosis regulator; Bax, BCL2 associated X; MAPK, mitogen-activated protein kinase; p, phosphorylated; TRAF6, TNF receptor-associated factor 6; TAK1, TGF- $\beta$-activated kinase 1; JNK, c-Jun N-terminal kinase. 
Table III. List of the 14 predicted target genes for miR-186.

\begin{tabular}{ll}
\hline Gene name & Gene Ensembl ID \\
\hline PSPH & ENSG00000146733 \\
YY1 & ENSG00000100811 \\
PABPC3 & ENSG00000151846 \\
DNM3 & ENSG00000197959 \\
CNTNAP1 & ENSG00000108797 \\
PNN & ENSG00000100941 \\
AHCYL1 & ENSG00000168710 \\
EP300 & ENSG00000100393 \\
NASP & ENSG00000132780 \\
PUM2 & ENSG00000055917 \\
SMAD6 & ENSG00000137834 \\
RBM26 & ENSG00000139746 \\
CETN2 & ENSG00000147400 \\
GPBP1 & ENSG00000062194 \\
\hline
\end{tabular}

SMAD6 was a genuine target of miR-186, a set of functional experiments was performed. First, miR-186 mimic was transfected into HUVECs, and SMAD6 expression was determined at both the mRNA and protein levels. The expression of SMAD6 was downregulated following miR-186 mimic overexpression in HUVECs (Fig. 3B and C). To further confirm that miR-186 could directly bind to SMAD6 and inhibit its expression, a luciferase reporter plasmid was generated with wild-type or mutant sequence of the SMAD6 predicted mRNA target fragment (Fig. 3D). The reporter constructs were co-transfected with miR-186 mimics or negative control oligonucleotides into HUVECs for $48 \mathrm{~h}$, and then luciferase activity was measured in the transfected cells. The results confirmed that the reporter construct with wild-type targeting sequence of SMAD6 mRNA caused a significant decrease in luciferase activity in cells transfected with miR-186, whereas the reporter construct with mutant sequence of SMAD6 produced no change in luciferase activity (Fig. 3E). These results suggested that miR-186 could bind to SMAD6 directly and inhibit its expression.

miR-186 enhances apoptosis in HUVECs by targeting SMAD6. The results demonstrated that overexpression of miR-186 induced apoptosis in HUVECs, and that SMAD6 was a direct target of miR-186. Therefore, it was hypothesized that miR-186 may affect the apoptosis of vascular endothelial cells through SMAD6. It has been reported that TGF stimulation induces cell apoptosis through the MAPK signaling pathway, while SMAD6 negatively regulates the TGF/MAPK signaling pathway $(29,30)$. Therefore, in the present study, first TGF- $\beta 1$ was used to induce cell apoptosis, and then the expression of SMAD6 was suppressed by miR-186. As expected, compared with the TGF- $\beta 1$-induced group, miR-186 significantly enhanced TGF- $\beta 1$-mediated cell apoptosis in HUVECs (Fig. 4A and B). In addition, miR-186 overexpression significantly enhanced the activation of the TGF/MAPK signaling pathways in HUVECs (Fig. 4C). Taken together, these results implied that miR-186 promoted the activation of MAPK signaling by targeting the inhibition of SMAD6, thus inducing endothelial cell apoptosis.
miR-186 has a role in KD-induced endothelial cell injury. The present study demonstrated that miR-186 was increased in HUVECs following culture with KD serum. It was also confirmed that miR-186 could promote HUVEC apoptosis. To demonstrate whether KD serum mediates endothelial cell apoptosis through miR-186, HUVECs were cultured with $20 \%$ serum either from patients with KD or healthy controls (the sera were from the patients listed in Table I). The results demonstrated that KD serum induced HUVEC apoptosis (Fig. 5A and B). However, KD serum-induced apoptosis was significantly inhibited by addition of the miR-186 inhibitor (30 nmol/1; Fig. 5A and B). In addition, in KD serum-treated HUVECs, the SMAD6 protein levels were decreased, and this effect was significantly reversed by addition of the miR-186 inhibitor (Fig. 5C). Similarly, that the results demonstrated that KD serum could induce activation of the TGF- $\beta$ /MAPK pathway in HUVECs, whereas addition of the miR-186 inhibitor significantly reversed the TGF- $\beta$ /MAPK pathway activation (Fig. 5D). The present results suggested that miR-186 in the serum of patients with KD may have an essential role in endothelial apoptosis by activating MAPK signaling through targeting of the SMAD6 gene.

\section{Discussion}

The present study demonstrated that overexpression of miR-186 may be a promoting factor for endothelial injury in KD. The results revealed that miR-186 was upregulated in KD serum and KD serum could increase miR-186 levels in HUVECs. Overexpression of miR-186 in HUVECs induced apoptosis, and the underlying mechanism likely involved the repression of SMAD6 and the subsequent activation of the TGF/MAPK pathway. In addition, the results demonstrated that KD serum induced HUVEC apoptosis through miR-186. The present study provides evidence that miR-186 may be a therapeutic target for KD.

miR-186 has an important role in a variety of diseases, especially in cancer. Hua et al (31) reported that miR-186 inhibited cell proliferation through targeting the oncogene GOLPH3 in prostate cancer. Liu et al (32) demonstrated that ectopic expression of miR-186 significantly inhibited cell growth in multiple myeloma. Notably, Wang et al (33) reported that circulating miR-186 could be considered a promising novel diagnostic biomarkers for the early phase of acute myocardial infarction. In the present study, miR-186 was demonstrated to inhibit expression of SMAD6 and activate MAPK signaling in HUVECs. In summary, the present results determined a new mechanism for miR-186 participating in the pathological process of KD.

$\mathrm{KD}$ is an important febrile illness causing multi-system vasculitis in childhood. The mechanisms involved in the pathogenesis of KD are not clearly understood and confirmatory diagnosis has not been established. Endothelial injury, an early risk marker of cardiovascular diseases, refers to loss of normal homeostatic function in blood vessels, and is characterized by inflammatory activity $(34,35)$. In KD, the long-term cardiovascular outcome for pediatric survivors is of important concern. Accelerated coronary atherosclerosis in KD-related lesions occurring in young adults has been reported and results in acute coronary syndrome or sudden death (36). Multiple 
A
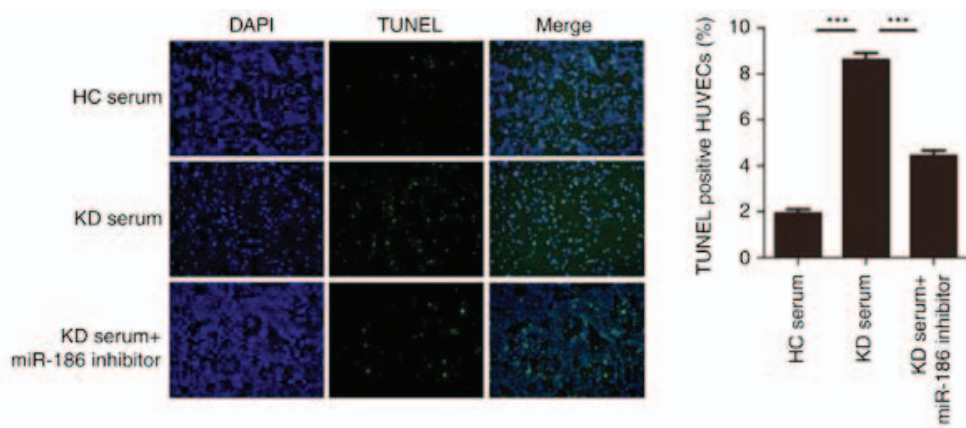

B
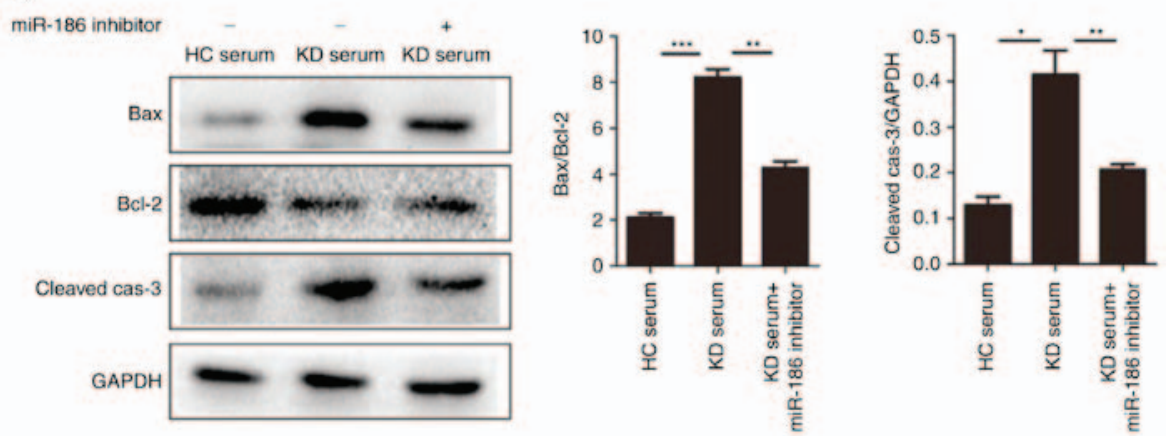

C
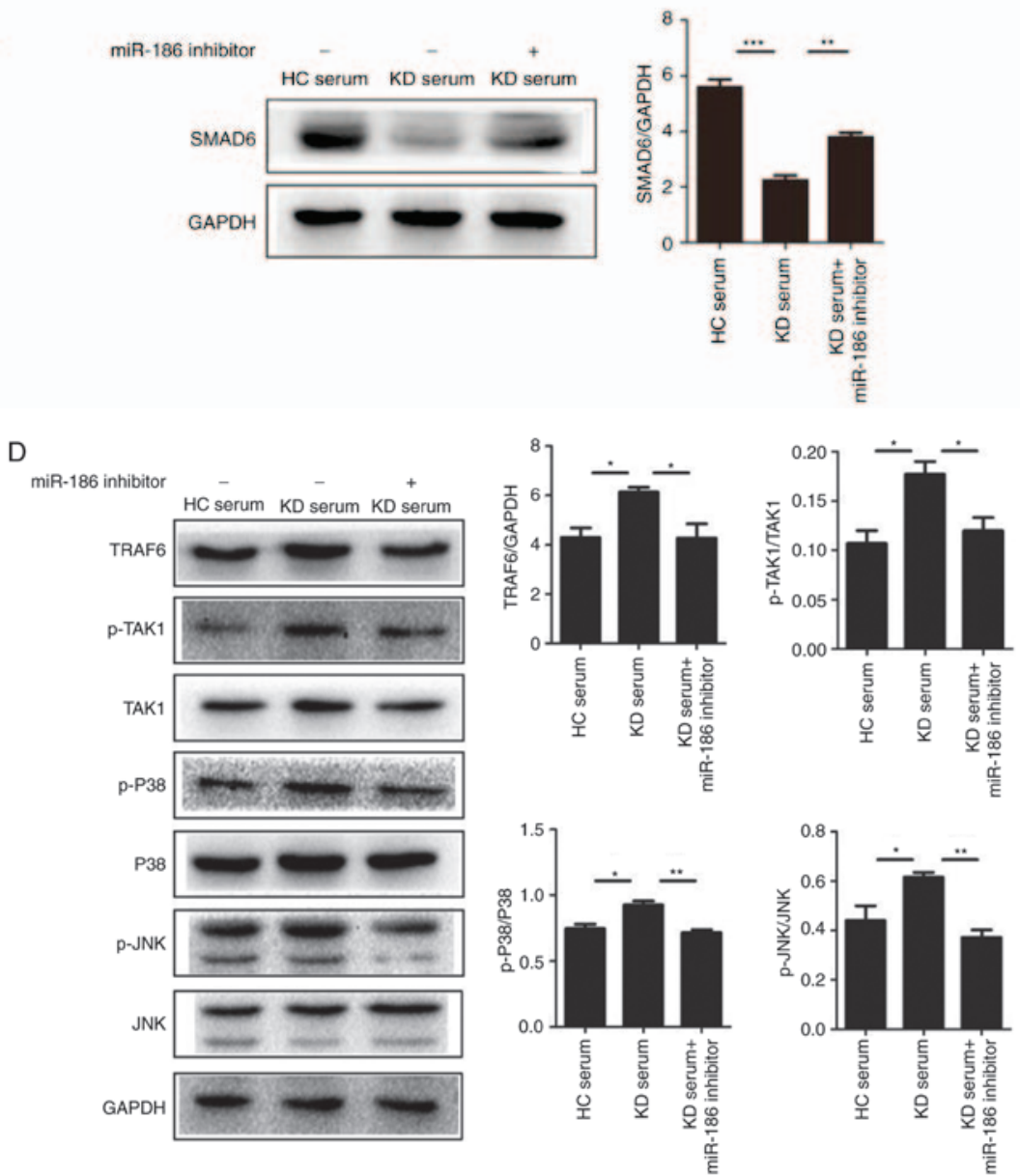

Figure 5. miR-186 promotes cell apoptosis induced by KD serum. (A) HUVEC apoptosis was induced by culture with KD serum and the effect of miR-186 was examined by the addition of the miR-186 inhibitor ( $30 \mathrm{nmol} / 1 ; \mathrm{n}=3$ ). Representative TUNEL images (magnification, $x 100)$ are shown. (B) Representative images and quantification from western blot analysis of the expression levels of apoptosis-related proteins. KD serum promoted the expression of apoptosis proteins, such as Bax/Bcl-2 ( $\mathrm{P}=0.000013)$ and cleaved-caspase-3 ( $\mathrm{P}=0.00221)$, while these effects were significantly blocked by the miR-186 inhibitor $(\mathrm{P}<0.05)$. (C) Representative images and quantification from western blot analysis of the SMAD6 protein expression levels in HUVECs induced by KD serum with $(\mathrm{P}=0.005023)$ or without $(\mathrm{P}=0.000079) \mathrm{miR}-186$ inhibitor. (D) KD serum alone activated the MAPK pathway, while KD serum with miR-186 inhibitor transfection significantly prevented this effect $(\mathrm{P}<0.05)$. Data are presented as the mean \pm standard error of the mean. ${ }^{*} \mathrm{P}<0.05,{ }^{* * *} \mathrm{P}<0.01$, and ${ }^{* * *} \mathrm{P}<0.001$, with comparisons indicated by lines. KD, Kawasaki disease; HUVECs, human umbilical vein endothelial cells; TUNEL, terminal deoxynucleotidyl transferase dUTP nick end labeling; Bcl-2, BCL2 apoptosis regulator; Bax, BCL2 associated X; SMAD6, SMAD family member 6; MAPK, mitogen-activated protein kinase; HC, healthy control; p, phosphorylated; TRAF6, TNF receptor associated factor 6; TAK1, TGF- $\beta$-activated kinase 1 ; JNK, c-Jun N-terminal kinase. 
studies exist on KD-associated endothelial injury; for example, the levels of blood cell-derived miR-223 in endothelial cells may act as a novel endocrine genetic signal and may participate in vascular injury of KD (18), while miR-125a-5p has been demonstrated to induce apoptosis in HUVECs through direct inhibition of MAP kinase kinase 7 levels resulting in Caspase-3 activation (37). However, the specific mechanisms of KD-induced endothelial injury remain unclear. The present study revealed that aberrantly expressed miR-186 in KD serum could induce endothelial cell apoptosis, which suggested that miR-186 could be the effector for impaired endothelium in KD and may be a new target for KD treatment.

TGF- $\beta$ is a multifunctional peptide that regulates proliferation, differentiation, apoptosis, and migration in many cell types (38). TGF- $\beta$-associated signal transduction involves SMAD-dependent and SMAD-independent pathways. The MAPK family of proteins consists of extracellular signal-regulated kinase (ERK), JNK/stress-activated protein kinase (SAPK) and p38 MAPK. The TGF- $\beta /$ MAPK pathway has been demonstrated to be involved in many processes in various diseases, including epithelial-myofibroblast transdifferentiation $(39,40)$, inflammatory (41) and apoptotic processes $(42,43)$. In endothelial cells, TGF- $\beta 1$ inhibits endothelial cell proliferation and migration (44), and induces endothelial cell apoptosis by inhibiting expression of the anti-apoptotic protein Bcl-2 and activating p38 MAPK (45). In $\mathrm{KD}$, a genetic association study has established that polymorphisms in the TGF- $\beta$ pathway influence disease susceptibility and coronary artery aneurysm formation (46). In addition, the TGF- $\beta$ related genes SMAD3, TGFB2, and TGFBR2 were observed to have a significant effect on KD susceptibility, severity, or treatment response. A later study provided more evidence on the role of the TGF- $\beta /$ SMAD3 signaling pathway in the arteritis of KD (47). In the present study, miR-186 was determined to directly target SMAD6 which then negatively regulated the TGF- $\beta$ /MAPK signaling pathway.

In conclusion, the present study confirmed that miR-186 was upregulated in serum from patients with acute KD and miR-186 upregulation appeared to be highly specific to the acute phase of KD. Furthermore, miR-186 was demonstrated to regulate the expression of SMAD6, which then negatively regulated TGF- $\beta$ signaling, resulting in feedback activation of the MAPK pathway to induce endothelial cell injury. These results suggest that miR-186 might be used as a target of KD treatment.

\section{Acknowledgements}

This study was supported by grants from the Zhejiang Provincial Medical and Health Science and Technology plan (grant no. WKJ-ZJ-1725), the Zhejiang Provincial Medical and Health Science and Technology plan (grant no. 2016KYB197), the Scientific Research Foundation of Wenzhou (grant no. Y20150015), and the Zhejiang Provincial Natural Science Foundation of China (grant no. Q15H020015).

\section{Competing interests}

The authors declare that they have no competing interests.

\section{References}

1. Newburger JW, Takahashi M and Burns JC: Kawasaki Disease. J Am Coll Cardiol 67: 1738-1749, 2016.

2. Gordon JB, Kahn AM and Burns JC: When children with Kawasaki disease grow up: Myocardial and vascular complications in adulthood. J Am Coll Cardiol 54: 1911-1920, 2009.

3. Kato H, Sugimura T, Akagi T, Sato N, Hashino K, Maeno Y, Kazue T, Eto G and Yamakawa R: Long-term consequences of Kawasaki disease. A 10- to 21-year follow-up study of 594 patients. Circulation 94: 1379-1385, 1996.

4. Newburger JW, Takahashi M, Beiser AS, Burns JC, Bastian J, Chung KJ, Colan SD, Duffy CE, Fulton DR, Glode MP, et al: A single intravenous infusion of gamma globulin as compared with four infusions in the treatment of acute Kawasaki syndrome. N Engl J Med 324: 1633-1639, 1991.

5. Leung DY, Cotran RS, Kurt-Jones E, Burns JC, Newburger JW and Pober JS: Endothelial cell activation and high interleukin-1 secretion in the pathogenesis of acute Kawasaki disease. Lancet 2: 1298-1302, 1989.

6. Amano S, Hazama F and Hamashima Y: Pathology of Kawasaki disease: I. Pathology and morphogenesis of the vascular changes. Jpn Circ J 43: 633-643, 1979.

7. Arkin A: A Clinical and pathological study of periarteritis nodosa: A report of five cases, one histologically healed. Am J Pathol 6: 401-426.5, 1930.

8. Shah V, Christov G, Mukasa T, Brogan KS, Wade A, Eleftheriou D, Levin M, Tulloh RM, Almeida B, Dillon MJ, et al: Cardiovascular status after Kawasaki disease in the UK. Heart 101: 1646-1655, 2015.

9. Tsuda E, Hamaoka K, Suzuki H, Sakazaki H, Murakami Y, Nakagawa M, Takasugi $\mathrm{H}$ and Yoshibayashi M: A survey of the 3-decade outcome for patients with giant aneurysms caused by Kawasaki disease. Am Heart J 167: 249-258, 2014.

10. Liang M: MicroRNA: A new entrance to the broad paradigm of systems molecular medicine. Physiol Genomics 38: 113-115, 2009.

11. Lagos-Quintana M, Rauhut R, Lendeckel W and Tuschl T: Identification of novel genes coding for small expressed RNAs. Science 294: 853-858, 2001.

12. Lewis BP, Burge CB and Bartel DP: Conserved seed pairing, often flanked by adenosines, indicates that thousands of human genes are microRNA targets. Cell 120: 15-20, 2005.

13. Yun KW, Lee JY, Yun SW, Lim IS and Choi ES: Elevated serum level of microRNA (miRNA)-200c and miRNA-371-5p in children with Kawasaki disease. Pediatr Cardiol 35: 745-752, 2014.

14. Shimizu C, Kim J, Stepanowsky P, Trinh C, Lau HD, Akers JC, Chen C, Kanegaye JT, Tremoulet A, Ohno-Machado L and Burns JC: Differential expression of miR-145 in children with Kawasaki disease. PLoS One 8: e58159, 2013.

15. Rowley AH, Pink AJ, Reindel R, Innocentini N, Baker SC, Shulman ST and Kim KY: A study of cardiovascular miRNA biomarkers for Kawasaki disease. Pediatr Infect Dis J 33: 1296-1299, 2014.

16. Rong X, Jia L, Hong L, Pan L, Xue X, Zhang C, Lu J, Jin Z, Qiu H, Wu R and Chu M: Serum miR-92a-3p as a new potential biomarker for diagnosis of Kawasaki disease with coronary artery lesions. J Cardiovasc Transl Res 10: 1-8, 2017.

17. He M, Chen Z, Martin M, Zhang J, Sangwung $P$, Woo B, Tremoulet AH, Shimizu C, Jain MK, Burns JC and Shyy JY: miR-483 targeting of CTGF suppresses endothelial-to-mesenchymal transition: Therapeutic implications in Kawasaki disease. Circ Res 120: 354-365, 2017.

18. Chu M, Wu R, Qin S, Hua W, Shan Z, Rong X, Zeng J, Hong L, Sun Y, Liu Y, et al: Bone marrow-derived microRNA-223 works as an endocrine genetic signal in vascular endothelial cells and participates in vascular injury from Kawasaki disease. J Am Heart Assoc 6: pii: e004878, 2017.

19. Cao C, Sun D, Zhang L and Song L: miR-186 affects the proliferation, invasion and migration of human gastric cancer by inhibition of Twist1. Oncotarget 7: 79956-79963, 2016.

20. Dong Y, Jin X, Sun Z, Zhao Y and Song X: MiR-186 inhibited migration of NSCLC via targeting cdc42 and effecting EMT process. Mol Cells 40: 195-201, 2017.

21. McCrindle BW, Rowley AH, Newburger JW, Burns JC, Bolger AF, Gewitz M, Baker AL, Jackson MA, Takahashi M, Shah PB, et al: Diagnosis, treatment, and long-term management of Kawasaki disease: A scientific statement for health professionals from the American heart association. Circulation 135: e927-e999, 2017. 
22. Livak KJ and Schmittgen TD: Analysis of relative gene expression data using real-time quantitative PCR and the $2^{-\Delta \Delta C_{\mathrm{T}}}$ method. Methods 25: 402-408, 2001.

23. Higashi K, Terai M, Hamada H, Honda T, Kanazawa $M$ and Kohno Y: Impairment of angiogenic activity in the serum from patients with coronary aneurysms due to Kawasaki disease. Circ J 71: 1052-1059, 2007.

24. Tian J, Lv HT, An XJ, Ling N and Xu F: Endothelial microparticles induce vascular endothelial cell injury in children with Kawasaki disease. Eur Rev Med Pharmacol Sci 20: 1814-1818, 2016.

25. He JH, Han ZP, Zou MX, Wang L, Lv YB, Zhou JB, Cao MR and Li YG: Analyzing the LncRNA, miRNA, and mRNA regulatory network in prostate cancer with bioinformatics software. J Comput Biol, 2017 (Epub ahead of print).

26. Yang JH, Li JH, Shao P, Zhou H, Chen YQ and Qu LH: starBase: A database for exploring microRNA-mRNA interaction maps from Argonaute CLIP-Seq and Degradome-Seq data. Nucleic Acids Res 39: D202-D209, 2011

27. Krek A, Grün D, Poy MN, Wolf R, Rosenberg L, Epstein EJ, MacMenamin P, da Piedade I, Gunsalus KC, Stoffel M and Rajewsky N: Combinatorial microRNA target predictions. Nat Genet 37: 495-500, 2005.

28. Vlachos IS, Paraskevopoulou MD, Karagkouni D, Georgakilas G, Vergoulis T, Kanellos I, Anastasopoulos IL, Maniou S, Karathanou K, Kalfakakou D, et al: DIANA-TarBase v7.0 Indexing more than half a million experimentally supported miRNA: mRNA interactions. Nucleic Acids Res 43: D153-D159, 2015.

29. Jung SM, Lee JH, Park J, Oh YS, Lee SK, Park JS, Lee YS, Kim JH, Lee JY, Bae YS, et al: Smad6 inhibits non-canonical TGF- $\beta 1$ signalling by recruiting the deubiquitinase A20 to TRAF6. Nat Commun 4: 2562, 2013.

30. Lee MK, Pardoux C, Hall MC, Lee PS, Warburton D, Qing J, Smith SM and Derynck R: TGF-beta activates Erk MAP kinase signalling through direct phosphorylation of ShcA. EMBO J 26: 3957-3967, 2007

31. Hua X, Xiao Y, Pan W, Li M, Huang X, Liao Z, Xian Q and Yu L: miR-186 inhibits cell proliferation of prostate cancer by targeting GOLPH3. Am J Cancer Res 6: 1650-1660, 2016.

32. Liu Z, Zhang G, Yu W, Gao N and Peng J: miR-186 inhibits cell proliferation in multiple myeloma by repressing Jagged1. Biochem Biophys Res Commun 469: 692-697, 2016.

33. Wang KJ, Zhao X, Liu YZ, Zeng QT, Mao XB, Li SN, Zhang M, Jiang C, Zhou Y, Qian C, et al: Circulating miR-19b-3p, miR-134-5p and miR-186-5p are promising novel biomarkers for early diagnosis of acute myocardial infarction. Cell Physiol Biochem 38: 1015-1029, 2016.

34. Davignon J and Ganz P: Role of endothelial dysfunction in atherosclerosis. Circulation 109 (23 Suppl 1): III27-III32, 2004.

35. Horio E, Kadomatsu T, Miyata K, Arai Y, Hosokawa K, Doi Y, Ninomiya T, Horiguchi H, Endo M, Tabata M, et al: Role of endothelial cell-derived angptl2 in vascular inflammation leading to endothelial dysfunction and atherosclerosis progression. Arterioscler Thromb Vasc Biol 34: 790-800, 2014

36. Hartopo AB and Setianto BY: Coronary artery sequel of Kawasaki disease in adulthood, a concern for internists and cardiologists. Acta Med Indones 45: 69-75, 2013.
37. Li Z, Jiang J, Tian L, Li X, Chen J, Li S, Li C and Yang Z: A plasma mir-125a-5p as a novel biomarker for Kawasaki disease and induces apoptosis in HUVECs. PLoS One 12: e0175407, 2017.

38. Ruiz-Ortega M, Rodriguez-Vita J, Sanchez-Lopez E, Carvajal G and Egido J: TGF-beta signaling in vascular fibrosis. Cardiovasc Res 74: 196-206, 2007.

39. Liu C, Chen F, Han X, Xu H and Wang Y: Role of TGF- $\beta 1 / p 38$ MAPK pathway in hepatitis B virus-induced tubular epithelial-myofibroblast transdifferentiation. Int J Clin Exp Pathol 7: 7923-7930, 2014.

40. Wei J, Li Z, Chen W, Ma C, Zhan F, Wu W and Peng Y: AEG-1 participates in TGF-betal-induced EMT through p38 MAPK activation. Cell Biol Int 37: 1016-1021, 2013.

41. Malik S, Suchal K, Khan SI, Bhatia J, Kishore K, Dinda AK and Arya DS: Apigenin ameliorates streptozotocin-induced diabetic nephropathy in rats via MAPK/NF- $\kappa \mathrm{B} / \mathrm{TNF}-\alpha$ and TGF- $\beta 1 /$ MAPK/fibronectin pathways. Am J Physiol Renal Physiol 2: F414-F422, 2017.

42. Hyman KM, Seghezzi G, Pintucci G, Stellari G, Kim JH, Grossi EA, Galloway AC and Mignatti P: Transforming growth factor-betal induces apoptosis in vascular endothelial cells by activation of mitogen-activated protein kinase. Surgery 132: 173-179, 2002.

43. Ferrari G, Terushkin V, Wolff MJ, Zhang X, Valacca C, Poggio $P$, Pintucci G and Mignatti P: TGF- $\beta 1$ induces endothelial cell apoptosis by shifting VEGF activation of p38 (MAPK) from the prosurvival $\mathrm{p} 38 \beta$ to proapoptotic $\mathrm{p} 38 \alpha$. Mol Cancer Res 10: 605-614, 2012.

44. Pollman MJ, Naumovski L and Gibbons GH: Vascular cell apoptosis: Cell type-specific modulation by transforming growth factor-beta1 in endothelial cells versus smooth muscle cells. Circulation 99: 2019-2026, 1999.

45. Tsukada T, Eguchi K, Migita K, Kawabe Y, Kawakami A, Matsuoka N, Takashima H, Mizokami A and Nagataki S: Transforming growth factor beta 1 induces apoptotic cell death in cultured human umbilical vein endothelial cells with down-regulated expression of bcl-2. Biochem Biophys Res Commun 210: 1076-1082, 1995.

46. Shimizu C, Jain S, Davila S, Hibberd ML, Lin KO, Molkara D, Frazer JR, Sun S, Baker AL, Newburger JW, et al: Transforming growth factor-beta signaling pathway in patients with Kawasaki disease. Circ Cardiovasc Genet 4: 16-25, 2011.

47. Shimizu C, Oharaseki T, Takahashi K, Kottek A, Franco A and Burns JC: The role of TGF- $\beta$ and myofibroblasts in the arteritis of Kawasaki disease. Hum Pathol 44: 189-198, 2013.

This work is licensed under a Creative Commons Attribution-NonCommercial-NoDerivatives 4.0 International (CC BY-NC-ND 4.0) License. 